\title{
Pedestal and Peak Structure in Jet Correlation
}

\author{
Charles B. Chiu ${ }^{1}$ and Rudolph C. Hwa ${ }^{2}$ \\ ${ }^{1}$ Center for Particle Physics and Department of Physics \\ University of Texas at Austin, Austin, TX 78712, USA \\ ${ }^{2}$ Institute of Theoretical Science and Department of Physics \\ University of Oregon, Eugene, OR 97403-5203, USA
}

\begin{abstract}
We study the characteristics of correlation between particles in jets produced in heavy-ion collisions. In the framework of parton recombination we calculate the $\eta$ and $\phi$ distributions of a pion associated with a trigger particle. The origin of the pedestal in $\Delta \eta$ is related to the longitudinal expansion of the thermal partons that are enhanced by the energy loss of hard partons traversing the bulk medium. The peaks in $\Delta \eta$ and $\Delta \phi$ are related to the same angular spread of the shower partons in a jet cone. No artificial short- or long-range correlations are put in by hand. A large part of the correlation between hadrons in jets is due to the correlation among the shower partons arising from momentum conservation. Recombination between thermal and shower partons dominates the correlation characterisitics in the intermediate $p_{T}$ region.

PACS numbers: 25.75.Gz, 25.75.-q
\end{abstract}

\section{Introduction}

The discovery of the medium effects on jets produced at the relativistic heavy-ion collider (RHIC) has contributed greatly to the understanding of the physics underlying hard partons traversing a hot and dense quark-gluon system[1,2]. Subsequently, a large number of experimental investigations have revealed details of the properties of jets, both on the near side and on the away side, in heavy-ion collisions (HIC) [3]-[8]. Particle correlations within jets are shown to have distinctive properties in the azimuthal angles $\phi$ and in pseudorapidities $\eta$ of the associated particles. Of particular noteworthiness is the peak in $\Delta \eta$ between the $\eta$ values of the trigger and the associated particles on the near side: it sits above a flat plateau or a pedestal [3]. No pedestal is found in $\Delta \phi$, although a peak in that variable is prominent. It is our aim in this paper to study the peaks and pedestal in $\Delta \phi$ and $\Delta \eta$ and to discuss the physical origins of these jet characteristics.

Our approach to the problem of correlation between particles in jets will be based on parton recombination that has been shown to be successful in reproducing the single-particle distributions in $\mathrm{Au}+\mathrm{Au}$ collisions [9], and in $\mathrm{d}+\mathrm{Au}$ collisions [10]. It has also been applied to the study of dihadron correlations $[11,12]$. However, since those investigations were carried out in the framework of the one-dimensional (1D) formulation of the recombination model, a generalization to account for the 3D geometry of the problem is necessary if we are to describe the features in the $\phi$ and $\eta$ variables. Once we consider the 3D aspects of jets 
the angular distribution of the shower partons in the jet cone becomes an important new feature. Our treatment will be phenomenological, since no results from perturbative QCD considerations can reliably be applied to the intermediate $p_{T}$ region, which is where the data on correlations are taken. Furthermore, the pedestal phenomenon suggests that the jet interacts with the environment in a way that is sensitive to the hydrodynamical expansion of the medium, since there is longitudinal expansion, but not azimuthal expansion. It turns out that we can relate the pedestal to the local enhancement of the thermal bath due to the energy loss of the hard parton to the medium.

Our approach to the problem, though not based on first principles, provides the first theoretical interpretation of the pedestal and peak structure of the jets produced at RHIC in quantitative terms. The roles that shower and thermal partons play in the hadronization process are found to be crucial and cannot be reinterpreted in any other model that we are aware of.

In Sec. 2 we describe how the 3D properties of jets are incorporated in the recombination formalism. In Sec. 3 the pedestal phenomenon is investigated, followed by the study of the peaks in $\Delta \eta$ and $\Delta \phi$ distributions in Sec. 4 . The conclusion is given in the last section.

\section{Two-Particle Distribution}

In the framework of parton recombination formulated in 1D for particle production in HIC [9]-[12], let us start by writing down the single-and two-particle distributions

$$
\begin{gathered}
\frac{d N_{\pi_{1}}}{p_{1} d p_{1}}=\frac{1}{p_{1}^{2}} \int \frac{d q_{1}}{q_{1}} \frac{d q_{2}}{q_{2}} F_{2}\left(q_{1}, q_{2}\right) R_{\pi_{1}}\left(q_{1}, q_{2}, p_{1}\right) \\
\frac{d N_{\pi_{1} \pi_{2}}}{p_{1} p_{2} d p_{1} d p_{2}}=\frac{1}{p_{1}^{2} p_{2}^{2}} \int\left(\prod_{j=1}^{4} \frac{d q_{j}}{q_{j}}\right) F_{4}\left(q_{1}, q_{2}, q_{3}, q_{4}\right) R_{\pi_{1}}\left(q_{1}, q_{3}, p_{1}\right) R_{\pi_{2}}\left(q_{2}, q_{4}, p_{2}\right),
\end{gathered}
$$

where the recombination function $(\mathrm{RF})$ for a pion is [13]

$$
R_{\pi}\left(q_{1}, q_{2}, p\right)=\frac{q_{1} q_{2}}{p^{2}} \delta\left(\frac{q_{1}}{p}+\frac{q_{2}}{p}-1\right) .
$$

The two- and four-parton distributions, $F_{2}$ and $F_{4}$, can be written in terms of their components as

$$
\begin{gathered}
F_{2}(1,2)=(\mathcal{T} \mathcal{T}+\mathcal{T S}+\mathcal{S S})_{12} \\
F_{4}(1,2,3,4)=(\mathcal{T} \mathcal{T}+\mathcal{T} \mathcal{S}+\mathcal{S S})_{13}(\mathcal{T} \mathcal{T}+\mathcal{T S}+\mathcal{S S})_{24}
\end{gathered}
$$

The thermal parton distribution is

$$
\mathcal{T}(q)=C q e^{-q / T}
$$


and the shower partons in a jet have the form

$$
\mathcal{S}(q)=\xi \sum_{i} \int d k k f_{i}(k) S_{i}^{j}(q / k)
$$

where $f_{i}(k)$ is the distribution of hard parton $i$ in a HIC, and $\xi$ is the average fraction of hard partons that emerge from the thermal medium to hadronize. $S_{i}^{j}$ is the shower-parton distribution (SPD) of parton $j$ in a shower initiated by a hard parton $i$; their parameterizations for various $i$ and $j$ are given in [14]. For the sake of clarity we have omitted the labels for parton species for $\mathcal{T}$ and $\mathcal{S}$ in the above equations and in what follows, except when the identity of a parton is needed for emphasis.

It is important to recognize that Eq. (7) should not be applied to every $\mathcal{S}$ that appears in Eqs. (4) and (5) because all the shower partons in $F_{2}$ and $F_{4}$ are created in the same jet, so all the $\mathcal{S} \mathcal{S} \cdots$ terms share the same hard parton with momentum $k$. Thus for two shower partons $(\mathcal{S S})$, their joint distribution is

$$
(\mathcal{S S})\left(q_{1}, q_{2}\right)=\xi \sum_{i} \int d k k f_{i}(k)\left\{S_{i}^{j}\left(\frac{q_{1}}{k}\right), S_{i}^{j^{\prime}}\left(\frac{q_{2}}{k-q_{1}}\right)\right\},
$$

where the quantity in the curly brackets has the form in terms of momentum fractions $x_{1}$ and $x_{2}$

$$
\left\{S_{i}^{j}\left(x_{1}\right), S_{i}^{j^{\prime}}\left(\frac{x_{2}}{1-x_{1}}\right)\right\}=\frac{1}{2}\left[S_{i}^{j}\left(x_{1}\right) S_{i}^{j^{\prime}}\left(\frac{x_{2}}{1-x_{1}}\right)+S_{i}^{j}\left(\frac{x_{1}}{1-x_{2}}\right) S_{i}^{j^{\prime}}\left(x_{2}\right)\right] .
$$

This is done to guarantee momentum conservation $x_{1}+x_{2} \leq 1$ and to symmetrize the order of emission of the two partons. Not only should Eq. (8) apply to two adjacent shower partons, like in $(\mathcal{S S})_{13}$ and $(\mathcal{S S})_{24}$, which recombine to form the pions at $p_{1}$ and $p_{2}$, respectively, but also to two shower partons like in $(\mathcal{T S})_{13}(\mathcal{T S})_{24}$, which do not recombine with each other, but separately with thermal partons. It is the structure in Eq. (8) that gives rise to correlation between two shower partons in a jet, and thereby endows the detected hadrons at high $p_{T}$ with correlation. However, that is sufficient primarily in the case of considering only the correlation among momentum magnitudes in 1D.

To generalize our consideration to $3 \mathrm{D}$, let us focus our attention first on the $(\mathcal{T S})$ components in Eq. (5) that give the most important contribution to the trigger and associated particles in central collision. To be specific let us further designate the trigger particle to be a $\pi^{+}$and the associated particle to be a $\pi^{-}$. The 3D expression for Eq. (2) in that case is

$$
\begin{aligned}
\frac{d N_{\pi^{+} \pi^{-}}}{p_{1} d p_{1} d \eta_{t r} d \phi_{t r} p_{2} d p_{2} d \eta d \phi}= & \frac{1}{\left(p_{1} p_{2}\right)^{2}} \xi \sum_{i} \int d k k f_{i}(k) \int\left(\prod_{j=1}^{4} d^{3} r_{j} \frac{d^{3} q_{j}}{q_{j}}\right) \\
& \times S_{i}^{u}\left(\vec{r}_{1}, \vec{q}_{1}\right) \mathcal{T}^{\bar{d}}\left(\vec{r}_{3}, \vec{q}_{3}\right) R_{\pi^{+}}^{(3)}\left(\vec{r}_{1}, \vec{r}_{3}, \vec{r}_{p_{1}} ; \vec{q}_{1}, \vec{q}_{3}, \vec{p}_{1}\right) \\
& \times S_{i}^{d}\left(\vec{r}_{2}, \vec{q}_{2}\right) \mathcal{T}^{\bar{u}}\left(\vec{r}_{4}, \vec{q}_{4}\right) R_{\pi^{-}}^{(3)}\left(\vec{r}_{2}, \vec{r}_{4}, \vec{r}_{p_{2}} ; \vec{q}_{2}, \vec{q}_{4}, \vec{p}_{2}\right)
\end{aligned}
$$

where $R^{(3)}$ is the $3 \mathrm{D}$ version of the RF and it includes the spatial coordinates. Since recombining partons must not only have collinear momenta but also have overlapping wave functions, there are many narrow Gaussian distributions in $R^{(3)}$ that reduce the phase space 
of integration. Let us first restrict our attention to the $x$ - $z$ plane, i.e. the plane containing the hard parton momentum $\vec{k}$ and the longitudinal direction $\hat{z}$, and assume that all $\vec{q}_{i}$ vectors are in that plane so that we may write

$$
\overrightarrow{q_{i}}=\left(q_{i}, \theta_{i}\right), \quad \vec{p}_{1}=\left(p_{1}, \theta_{\text {trig }}\right), \quad \vec{p}_{2}=\left(p_{2}, \theta\right)
$$

and

$$
\eta_{i}=\ln \cot \frac{\theta_{i}}{2}, \quad \eta_{\text {trig }}=\ln \cot \frac{\theta_{\text {trig }}}{2}, \quad \eta=\ln \cot \frac{\theta}{2}
$$

We further assume that $\vec{q}_{1}$ is in the direction of $\vec{k}$ and that the trigger momentum $\vec{p}_{1}$ is also along $\vec{k}$. This is a simplification that does not compromise the angular correlation between the trigger and associated particles, which we shall study in detail. Since $R^{(3)}$ requires the wave functions of the recombining partons to overlap, the shower and thermal partons at $\vec{r}_{1}$ and $\vec{r}_{3}$ should be nearby. These assumptions allow us to reduce the trigger momentum part of Eq. (10) to the usual 1D formulation

$$
\begin{array}{r}
\int d^{3} r_{1} d^{3} r_{3} \frac{d^{3} q_{1}}{q_{1}} \frac{d^{3} q_{3}}{q_{3}} S_{i}^{u}\left(\vec{r}_{1}, \vec{q}_{1}\right) \mathcal{T}^{\bar{d}}\left(\vec{r}_{3}, \vec{q}_{3}\right) R_{\pi^{+}}^{(3)}\left(\vec{r}_{1}, \vec{r}_{3}, \vec{r}_{p_{1}} ; \vec{q}_{1}, \vec{q}_{3}, \vec{p}_{1}\right) \\
=\left.\int \frac{d q_{1}}{q_{1}} \frac{d q_{3}}{q_{3}} S_{i}^{u}\left(\frac{q_{1}}{k}\right) \mathcal{T}\left(q_{3}, \theta_{3}\right)\right|_{\theta_{3}=\theta_{1}=\theta_{\text {trig }}} R_{\pi^{+}}^{(1)}\left(q_{1}, q_{3}, p_{1}\right)
\end{array}
$$

where $R^{(1)}$ is the usual RF in 1D, given in Eq. (3). The superscript $\bar{d}$ on $\mathcal{T}$ is omitted on the RHS since the flavor dependence of the thermal partons is negligible and ignored. We shall assume that at midrapidity, $-0.7<\eta_{\text {trig }}<+0.7$, the thermal distribution does not contain essential dependence on $\eta_{3}$ so that the condition $\theta_{3}=\theta_{1}=\theta_{\text {trig }}$ does not lead to any restriction that would cause $\mathcal{T}\left(q_{3}, \theta_{3}\right)$ to deviate from the usual parameterization given in Eq. (6).

In the case of the associated particle we must recognize that the shower parton $\vec{q}_{2}$ may be emitted at an angle $\psi$ relative to the hard parton momentum $\vec{k}$. Since $\vec{k}$ forms an angle $\theta_{1}$ with the $z$ axis (after identifying the direction $\hat{q}_{1}$ with $\hat{k}$ ), we have

$$
\psi=\theta_{2}-\theta_{1} .
$$

The recombination of the shower parton at $\vec{q}_{2}$ with a thermal parton at $\vec{q}_{4}$ requires that they are not only collinear, but also overlapping spatially. There are therefore $\delta$-functions, $\delta\left(\theta_{2}-\theta_{4}\right) \delta\left(\frac{\theta_{2}+\theta_{4}}{2}-\theta\right)$, that force the momentum $\vec{p}_{2}$ of the associated particle to be in the same direction as $\hat{q}_{2}$ and $\hat{q}_{4}$. The corresponding $3 \mathrm{D}$ integrations result in

$$
\begin{aligned}
& \int d^{3} r_{2} d^{3} r_{4} \frac{d^{3} q_{2}}{q_{2}} \frac{d^{3} q_{4}}{q_{4}} S_{i}^{d}\left(\vec{r}_{2}, \vec{q}_{2}\right) \mathcal{T}^{\bar{u}}\left(\vec{r}_{4}, \vec{q}_{4}\right) R_{\pi^{-}}^{(3)}\left(\vec{r}_{2}, \vec{r}_{4}, \vec{r}_{p_{2}} ; \vec{q}_{2}, \vec{q}_{4}, \vec{p}_{2}\right) \\
& =\left.\int \frac{d q_{2}}{q_{2}} \frac{d q_{4}}{q_{4}} S_{i}^{d}\left(\frac{q_{2}}{k-q_{1}}, \psi\right) \mathcal{T}\left(q_{4}, \theta_{4}\right)\right|_{\theta_{4}=\theta_{2}=\theta_{1}+\psi} R_{\pi^{-}}^{(1)}\left(q_{2}, q_{4}, p_{2}\right)
\end{aligned}
$$

This equation is very similar to Eq. (13) but with one crucial difference; that is, the shower parton distribution now depends on $\psi$. In Eq. (13) no angular dependence of the shower 
parton $\vec{q}_{1}$ is assumed for simplicity, since it is only the difference between $\vec{q}_{1}$ and $\overrightarrow{q_{2}}$ that matters. That angular difference, $\psi$, made explicit in Eq. (14), is translated into the angular difference between $\vec{p}_{1}$ and $\vec{p}_{2}$. We have tacitly assumed that the thermal partons have local angular spread that allows a $\overrightarrow{q_{3}}$ to recombine with $\vec{q}_{1}$ to form $\vec{p}_{1}$, and similarly $\vec{q}_{4}$ with $\vec{q}_{2}$ to form $\vec{p}_{2}$, in such a way that $\vec{p}_{1}$ and $\vec{p}_{2}$ are in the directions of $\vec{q}_{1}$ and $\vec{q}_{2}$, respectively.

The dependence of a SPD on $\psi$ cannot be determined from pQCD, since the values of $p_{T}$ concerned is in the $1<p_{T}<4 \mathrm{GeV} / \mathrm{c}$ range. We shall assume that the SPD in Eq. (15) can be written in the following factorizable form

$$
S_{i}^{d}\left(\frac{q_{2}}{k-q_{1}}, \psi\right)=S_{i}^{d}\left(\frac{q_{2}}{k-q_{1}}\right) G\left(\psi, \frac{q_{2}}{k}\right),
$$

where the dependence on $\psi$ has a Gaussian distribution

$$
G(\psi, x)=\exp \left[-\frac{\psi^{2}}{2 \sigma^{2}(x)}\right],
$$

whose half-width depends on the momentum fraction $x$ of the shower parton

$$
\sigma(x)=\sigma_{0}(1-x)
$$

with $\sigma_{0}$ being a parameter that is adjustable. $S_{i}^{d}$ on the RHS of Eq. (16) is to be symmetrized with $S_{i}^{u}$ in Eq. (13) without further complication from angular consideration. The momentum fraction relevant for $\sigma(x)$ is $q_{2} / k$, independent of $q_{1}$. Once we obtain Eq. (16) where $G\left(\psi, q_{2} / k\right)$ stands as a modifying factor, it is reasonable to liberate that factor from the way in which it is derived, and allow it to assume a $3 \mathrm{D}$ property so that when we later consider the $\Delta \phi$ behavior in the transverse plane the same factor $G\left(\psi, q_{2} / k\right)$ applies to the azimuthal angle between $\vec{p}_{1}$ and $\vec{p}_{2}$ with $\psi$ replaced by $\Delta \phi$. Using Eqs. (12) and (14) we obtain with $\theta=\theta_{2}$

$$
\tan \frac{\psi}{2}=\tan \frac{\theta-\theta_{1}}{2}=g\left(\eta, \eta_{1}\right)=\frac{e^{-\eta}-e^{-\eta_{1}}}{1+e^{-\eta-\eta_{1}}}
$$

where $\eta_{1}$ is to be identified with $\eta_{\text {trig }}$.

We now can write Eq. (10) with the help of Eqs. (3), (13) and (15) in the simpler form

$$
\begin{array}{r}
\frac{d N_{\pi^{+} \pi^{-}}^{T S T S}}{p_{1} d p_{1} d \eta_{1} d \phi_{1} p_{2} d p_{2} d \eta d \phi}=\frac{1}{\left(p_{1} p_{2}\right)^{3}} \xi \sum_{i} \int d k k f_{i}(k) \int d q_{1} \int d q_{2} \theta\left(k-q_{1}-q_{2}\right) \\
\sum_{j, j^{\prime}} \mathcal{T}\left(p_{1}-q_{1}, \eta_{1}\right)\left\{S_{i}^{j}\left(\frac{q_{1}}{k}\right), S_{i}^{j^{\prime}}\left(\frac{q_{2}}{k-q_{1}}\right)\right\} \mathcal{T}\left(p_{2}-q_{2}, \eta\right) \\
\left.G\left(\psi, \frac{q_{2}}{k}\right)\right|_{\psi=2 \tan ^{-1} g\left(\eta, \eta_{1}\right)}
\end{array}
$$

where $i$ is summed over all hard parton species. If $i$ is a valence quark of $\pi^{+}$or $\pi^{-}$, the corresponding SPD is $K$, while if $i$ is a sea quark, the corresponding SPD is $L$ in the notation of [14], where $K=S_{i}^{v a l+s e a}$ and $L=S_{i}^{\text {sea }}$. The integral over $k$ will, in practice, be from 3 to $30 \mathrm{GeV} / \mathrm{c}$, and $q_{i}$ will be integrated from 0 to $p_{i}$, although the formalism is not reliable for any transverse momentum less than $1 \mathrm{GeV} / \mathrm{c}$. There are other terms of $F_{4}$ contained in Eq. (5) that can be written out as in Eq. (20), but will not be detailed here. 


\section{Pedestal and Background Subtraction}

The experimental procedure of making background subtraction involves multiple considerations, and the result is that there remains a residual pedestal in $\Delta \eta$ in central collisions, but no pedestal in $\Delta \phi$, where $\Delta \eta$ and $\Delta \phi$ are differences in $\eta$ and $\phi$ between the associated particle and the trigger. In [11] the dihadron correlation is calculated with the assumption that the factorizable part of the two-particle distribution corresponds to the background. That leaves only the $(\mathcal{T S}+\mathcal{S S})_{13}(\mathcal{T S}+\mathcal{S S})_{24}$ terms of $F_{4}$ to consider. We now find that in order to understand the origin of the pedestal what constitutes the background must be reconsidered.

The first point that we want to address is the effect of energy loss of hard partons traversing the hot medium. The parameter $\xi$ in Eqs. (7) and (8), being only 0.07 as determined in [9] for central $\mathrm{Au}+\mathrm{Au}$ collisions, implies that a large fraction of the hard partons produced are absorbed or attenuated by the bulk medium. Those that emerge to hadronize outside must have been created near the surface. In the short distance that such a parton travels in the medium it must on average lose some energy and locally enhance the thermal motion of the partons in the environment. Those enhanced thermal partons should have an effective inverse slope $T$ that is slightly higher (by $\Delta T$ ) than that of the usual thermal partons not influenced by the passage of any hard partons. The latter corresponds to what is measured for the low- $p_{T}$ pions in single-particle distribution, which for $p_{T}<2 \mathrm{GeV} / \mathrm{c}$ has led to the determination of $T=0.317 \mathrm{GeV} / \mathrm{c}$ in [9]. If $\Delta T$ is small, as we expect it to be, it does not make too much difference in the calculation of the single-particle distribution in the intermediate $p_{T}$ region. However, in principle, in thermal-shower recombination the shower partons recombine with the enhanced thermal partons that are in the immediate vicinity of the hard parton that creates the shower. To distinguish the two values of the inverse slope, let us use $T$ to denote the one for the enhanced thermal parton, since that is the only physically relevant one for $\mathcal{T S}$ recombination, i.e., it is what appears in Eq. (6) for $\mathcal{T}$. Let us use $T_{0}$ to denote the inverse slope for the thermal medium undisturbed by jet quenching, i.e., $T_{0}=0.317 \mathrm{GeV} /$ c. We define

$$
\Delta T=T-T_{0},
$$

which is a parameter to be determined phenomenologically in this paper, although it can presumably be determined directly by dedicated experimentation. We shall relate $\Delta T$ to the pedestal. The physics involved is sensible, since the pedestal is seen only for central collisions where $\mathcal{T S}$ recombination is important. In peripheral collisions thermal partons play a minor role and there are few of them to be enhanced.

We now give an argument why the pedestal is seen in $\Delta \eta$, but not in $\Delta \phi$. When a hard parton is first scattered or created at a large angle relative to the incident beam direction, the bulk medium is in a highly compressed state. As it expands longitudinally, the region of enhanced thermal partons expands with the whole system, although limited to the neighborhood of where the hard parton traverses the thin layer close to the surface. Thus a section at midrapidity can have the enhanced $T$. In the transverse plane, on the other hand, the expansion is in the radial direction only; that is, there is no mixing across different azimuthal sections. The subtraction scheme carried out by STAR [3] defines the yield $d N / d \Delta \phi$ to be zero at $|\Delta \phi|=1$ after subtraction. Since there is no expansion in the $\phi$ direction, we can 
take this subtraction scheme to imply that all thermal partons that are enhanced stay inside the $|\Delta \phi|<1$ region, and that for $|\Delta \phi|>1$ there is no thermal-thermal recombination that is not in the background. There may still be an enhancement of the yield inside the $|\Delta \phi|<1$ region that plays a role similar to the pedestal in $\Delta \eta$, but it cannot extend beyond $|\Delta \phi|=1$ by virtue of the subtraction scheme.

The above description cannot be demonstrated by a transport model, since we do not have a Monte Carlo code for the evolution process. However, the physical content of our discussion will be embodied in our quantitative formulation below. The recombination model actually provides a more transparent description of the hadronization process than what a code without reliable equations can offer.

Let us first consider the particles that are associated with a trigger. Since in $\mathrm{Au}+\mathrm{Au}$ collisions the $\mathcal{T S}$ component is dominant in the intermediate $p_{T}$ region [9], which includes the trigger window $4<p_{T}<6 \mathrm{GeV} / \mathrm{c}$, we select the $(\mathcal{T S})_{13}$ term in Eq. (5) for our discussion below, although both terms in $(\mathcal{T S}+\mathcal{S S})_{13}$ are included in our calculation. Thus the parts of $F_{4}$ that contribute to the trigger and its associated particles are

$$
F_{4}^{\text {tr+as }}=(\mathcal{T S})_{13}(\mathcal{T} \mathcal{T}+\mathcal{T S}+\mathcal{S S})_{24}
$$

Among the three terms on the right side it is clear that $(\mathcal{T S}+\mathcal{S S})_{24}$ are directly related to the jet and contribute to the peak in the associated particle distribution (APD), while $(\mathcal{T} \mathcal{T})_{24}$ does not involve the shower partons but can nevertheless contribute to the APD outside the peak. It is therefore our prime candidate for the pedestal. The absence of an obvious pedestal in the APD in $\Delta \phi$ is a consequence of the background subtraction scheme in the analysis of the experimental data, where the $\Delta \phi$ distribution at $|\Delta \phi|=1$ is identified to be the background. In our description of the APD the background corresponds to

$$
F_{4}^{b g}=(\mathcal{T S})_{13}\left(\mathcal{T}_{0} \mathcal{T}_{0}\right)_{24}
$$

where $\mathcal{T}_{0}(q)$ is the thermal parton distribution in the absence of any hard partons, i.e.,

$$
\mathcal{T}_{0}(q)=C q e^{-q / T_{0}}
$$

The meaning of $T_{0}$ has already been discussed in connection with Eq. (21). We have tacitly assumed that the normalization factor $C$ is unchanged from that in Eq. (6). The experimental subtraction scheme implies that

$$
(\mathcal{T S})_{13}(\mathcal{T} \mathcal{T})_{24}=(\mathcal{T S})_{13}\left(\mathcal{T}_{0} \mathcal{T}_{0}\right)_{24}
$$

at $|\Delta \phi|=1$. We now define the APD with background subtracted to be generated by

$$
F_{4}^{A P}=(\mathcal{T S})_{13}\left(\mathcal{T} \mathcal{T}-\mathcal{T}_{0} \mathcal{T}_{0}+\mathcal{T S}+\mathcal{S S}\right)_{24}
$$

in which $\left(\mathcal{T} \mathcal{T}-\mathcal{T}_{0} \mathcal{T}_{0}\right)_{24}$ vanishes at $|\Delta \phi|=1$, but need not be zero at $|\Delta \phi|<1$. Equation (26) is our basic input in the calculation of the APD.

Let us now write our two-particle distribution in a form suitable for application of the experimental cuts. In Eq. (20) we have a differential distribution in $d p_{1} d \eta_{1} d \phi_{1} d p_{2} d \eta d \phi$ where 
$\eta_{1}$ and $\phi_{1}$ denote the variables of the trigger, and $\eta$ and $\phi$ those of the associated particle. With the definition

$$
\Delta \eta=\eta-\eta_{1}, \quad \Delta \phi=\phi-\phi_{1},
$$

we change the differentiated variables to $d p_{1} d p_{2} d \eta_{1} d \Delta \eta d \Delta \phi$, with $\phi_{1}$ being used as a free reference point in the assemblage of the $\Delta \phi$ distribution; the trigger $\eta_{1}$ is to be integrated over the trigger window $-0.7<\eta_{1}<+0.7$. We then have

$$
\begin{aligned}
N(1,2) & \equiv \frac{d N_{\pi_{1} \pi_{2}}}{d p_{1} d p_{2} d \eta_{1} d \Delta \eta d \Delta \phi} \\
& =\frac{1}{p_{1} p_{2}} \int\left(\prod_{j=1}^{4} \frac{d q_{j}}{q_{j}}\right) F_{4}^{A P}\left(q_{1}, q_{2}, q_{3}, q_{4}\right) R_{\pi_{1}}\left(q_{1}, q_{3}, p_{1}\right) R_{\pi_{2}}\left(q_{2}, q_{4}, p_{2}\right) .
\end{aligned}
$$

The experimental APD involves integrations over the trigger window in $\eta_{1}$ and $p_{1}$ and the window of the associated particle in $p_{2}$. It is

$$
\frac{d N^{A P}}{d \Delta \eta d \Delta \phi}=\frac{\int_{p_{a}}^{p_{b}} d p_{2} \int_{4}^{6} d p_{1} \int_{-0.7}^{0.7} d \eta_{1} N(1,2)}{\int_{4}^{6} d p_{1} \int_{-0.7}^{0.7} d \eta_{1} N(1)}
$$

where $N(1)$ is the one-particle trigger distribution that receives contribution from the $(\mathcal{T S})_{13}$ component only, i.e.

$$
N(1) \equiv \frac{d N_{\pi_{1}}^{t r}}{d p_{1} d \eta_{1}}=\frac{1}{p_{1}^{2}} \xi \sum_{i} \int d k k f_{i}(k) d q_{1} \sum_{j} \mathcal{T}\left(p_{1}-q_{1}, \eta_{1}\right) S_{i}^{j}\left(\frac{q_{1}}{k}\right)
$$

The two-particle distribution $N(1,2)$ in Eq. (29) can be divided into two explicit pieces. For the remainder of this section we consider only the piece related to the pedestal

$$
\begin{aligned}
N(1,2)^{p e d} \equiv & \frac{d N_{\pi_{1} \pi_{2}}^{p e d}}{d p_{1} d p_{2} d \eta_{1} d \Delta \eta d \Delta \phi} \\
= & \frac{1}{\left(p_{1} p_{2}\right)^{2}} \xi \sum_{i} \int d k k f_{i}(k) d q_{1} d q_{2} \\
& \sum_{j} \mathcal{T}\left(p_{1}-q_{1}, \eta_{1}\right) S_{i}^{j}\left(\frac{q_{1}}{k}\right)\left[\mathcal{T}\left(q_{2}, \Delta \eta\right) \mathcal{T}\left(p_{2}-q_{2}, \Delta \eta\right) H(\Delta \phi)\right. \\
& \left.-\mathcal{T}_{0}\left(q_{2}\right) \mathcal{T}_{0}\left(p_{2}-q_{2}\right)\right]
\end{aligned}
$$

where we have extracted the $\phi$ dependence of the associated particle in the form of a factor $H(\Delta \phi)$, which satisfies some constraints to be specified below. It should be noted that Eq. (31) is factorizable, since the two parts of $(\mathcal{T S})_{13}\left(\mathcal{T} \mathcal{T}-\mathcal{T}_{0} \mathcal{T}_{0}\right)_{24}$ are independent of each other. The $(\mathcal{T S})_{13}$ part contributes to the trigger distribution given in Eq. (30). It will be cancelled in the ratio defined in Eq. (29).

Using Eq. (24), the integration of the last term in Eq. (31) over $q_{2}$ can readily be carried out, giving

$$
\frac{1}{p_{2}^{3}} \int_{0}^{p_{2}} d q_{2} \mathcal{T}_{0}\left(q_{2}\right) \mathcal{T}_{0}\left(p_{2}-q_{2}\right)=\frac{C^{2}}{6} \exp \left(-p_{2} / T_{0}\right)
$$


which, upon further integration over the window $\left(p_{a}, p_{b}\right)$ of the associated particle, results in

$$
\int_{p_{a}}^{p_{b}} d p_{2} p_{2} \frac{d N^{b g}}{p_{2} d p_{2}}=\frac{1}{6}\left(C T_{0}\right)^{2} h\left(\frac{p_{a}}{T_{0}}, \frac{p_{b}}{T_{0}}\right),
$$

where

$$
h(x, y)=(1+x) e^{-x}-(1+y) e^{-y} .
$$

Similar result follows for the $\mathcal{T} \mathcal{T}$ term in Eq. (31).

Next, we consider the $\Delta \eta$ and $\Delta \phi$ dependences of the pedestal in Eq. (31). As we have discussed qualitatively in the beginning of this section, the longitudinal expansion allows the enhanced thermal partons to extend over a wide range in $\eta$, but radial expansion does not increase the range in $\phi$. Putting these properties into quantitative terms, we give $\mathcal{T}\left(q_{2}\right)$ no essential dependence on $\Delta \eta$ in Eq. (31), but a Gaussian dependence on $\Delta \phi$ so that

$$
H(\Delta \phi)=c \exp \left(-\Delta \phi^{2} / 2 \sigma_{\phi}^{2}\right)
$$

The normalization $c$ is to be determined by the condition that when $\Delta \phi$ is integrated over the experimental window $(-0.5,+0.5)$ we obtain

$$
\int_{-0.5}^{0.5} d \Delta \phi H(\Delta \phi)=1
$$

In this way we can relate Eq. (31) to the pedestal observed in the experiment without explicit factors dependent on the experimental windows. The half-width $\sigma_{\phi}$ is adjusted so that the quantity inside the square brackets in Eq. (31) vanishes at $|\Delta \phi|=1$, as required by the subtraction scheme, Eq. (25). What we do here is to fix all the extra free parameters of the problem by the experimental features of the data. Since our aim is to reproduce the observed characteristics of the data, which are presented with specific cuts, it is impossible to do so without incorporating those cuts. However, it does not imply that we are merely fitting the data with free parameters. We shall perform several multi-dimensional integrals to obtain in the next section the APD with the peak whose magnitude is a prediction of our model calculation.

Upon integration of Eq. (29) over $\Delta \phi$ in the acceptance window, we get for the pedestal part

$$
\frac{d N^{p e d}}{d \Delta \eta}=\int_{-0.5}^{0,5} d \Delta \phi \frac{d N^{p e d}}{d \Delta \eta d \Delta \phi}=\frac{C^{2}}{6}\left[T^{2} h\left(\frac{p_{a}}{T}, \frac{p_{b}}{T}\right)-T_{0}^{2} h\left(\frac{p_{a}}{T_{0}}, \frac{p_{b}}{T_{0}}\right)\right],
$$

where we have made use of the factorizability of Eq. (31) and the result of integration over $p_{2}$ given in Eq. (33). This is the constant pedestal in $\Delta \eta$, which we relate to $\Delta T$. Using $C=23.2(\mathrm{GeV} / \mathrm{c})^{-1}, T_{0}=0.317 \mathrm{GeV} / \mathrm{c}$ from $[9], p_{a}=2, p_{b}=4 \mathrm{GeV} / \mathrm{c}$ as in the experiment [3], and Eq. (21) for the definition of $\Delta T$, we fit the pedestal height of $\sim 0.05$ [3] and obtain

$$
\Delta T=15 \mathrm{MeV}
$$

Thus the inverse slope of the enhanced thermal partons is only $5 \%$ higher than that of the un-enhanced partons, small enough to have a negligible effect on the calculation of the singleparticle distribution using $T_{0}$. However, the effect on the associated particle distribution is 
evidently not negligible. Although Eq. (38) is a fitted result, it should be emphasized that it is in the specific model of parton recombination that the pedestal phenomenon is interpreted as a consequence of the enhancement of the thermal energy of the soft partons due to energy loss of the hard parton.

Equation (37) is the projection of $d N^{A P} / d \Delta \eta d \Delta \phi$ onto $\Delta \eta$. We now project it onto $\Delta \phi$ by integrating it over $\Delta \eta$ from -1 to +1 in accordance to the experimental cut [3] and obtain from Eq. (31) for the pedestal part

$$
\frac{d N^{p e d}}{d \Delta \phi}=\int_{-1}^{1} d \Delta \eta \frac{d N^{p e d}}{d \Delta \eta d \Delta \phi}=\frac{C^{2}}{3}\left[T^{2} h\left(\frac{p_{a}}{T}, \frac{p_{b}}{T}\right) H(\Delta \phi)-T_{0}^{2} h\left(\frac{p_{a}}{T_{0}}, \frac{p_{b}}{T_{0}}\right)\right] .
$$

This is not a flat pedestal as in the case of $\Delta \eta$, but is nevertheless a remnant of the effect of enhanced thermal partons sitting under a peak from the $(\mathcal{T S}+\mathcal{S S})_{24}$ part of $F_{4}^{A P}$ that we have not yet calculated. Although it is not visible as a plateau, its origin is the same as that which gives rise to the pedestal in $\Delta \eta$. It is the background subtraction in $\Delta \phi$ that forces Eq. (39) to vanish at $|\Delta \phi|=1$, which is achieved by our choice of $\sigma_{\phi}$ in Eq. (35) its value is

$$
\sigma_{\phi}=1.2
$$

With this choice of $\sigma_{\phi}$ Eq. (39) is entirely specified numerically. The $\Delta \phi$ dependence of $d N^{\text {ped }} / d \Delta \phi$ is a broad mount between $\Delta \phi= \pm 1$, which we shall exhibit later together with the complete $d N / d \Delta \phi$. This mount is the projection of a ridge in the 3D display of APD in $(\Delta \eta, \Delta \phi)$ onto the $\Delta \phi$ subspace.

This completes our discussion of the $\left(\mathcal{T} \mathcal{T}-\mathcal{T}_{0} \mathcal{T}_{0}\right)$ contribution to $F_{4}^{A P}$ in Eq. (26) and then to Eqs. (28) and (29). We now proceed to the last two terms of $F_{4}^{A P}$.

\section{$4 \quad$ Peaks in $\Delta \eta$ and $\Delta \phi$}

We now return to Eq. $(26)$ and consider the $(\mathcal{T S}+\mathcal{S S})_{24}$ terms in $F_{4}^{A P}$ that we have put aside. The $\mathcal{T S}$ component has already been described in detail in Eq. (20). The $\mathcal{S S}$ component is less important except in peripheral collisions. It can be included by the replacement of the $\left\{S_{i}^{j}, S_{i}^{j^{\prime}}\right\} \mathcal{T}$ term in Eq. (20) by a $\left\{S_{i}^{j}, S_{i}^{j^{\prime}}, S_{i}^{j^{\prime \prime}}\right\}$ term with appropriate symmetrization. However, since the recombination of two shower partons reproduces the fragmentation function in accordance to

$$
x D_{i}^{\pi}(x)=\int \frac{d x_{1}}{x_{1}} \frac{d x_{2}}{x_{2}}\left\{S_{i}^{j}\left(x_{1}\right), S_{i}^{j^{\prime}}\left(\frac{x_{2}}{1-x_{1}}\right)\right\} R_{\pi}\left(x_{1}, x_{2}, x\right)
$$

from which the SPDs are derived in the first place, we may replace a $S S R$ term by a $x D$ term. For notational simplicity we leave the $(\mathcal{S S})_{24}$ terms out in our description below, but numerically include their recombination in the calculated result.

Let us recall that starting from the general formula Eq. (10) we first focussed on the variables in the plane containing $\vec{k}$ and the longitudinal direction $\hat{z}$ and related the relevant angles to pseudorapidities in Eq. (12). We then defined the angle $\psi$ between the shower parton $\vec{q}_{2}$ and the hard parton $\vec{k}$ to be as given in Eq. (14). The angular dependence of the SPD is then expressed by a Gaussian distribution $G(\psi, x)$ in Eq. (17), put in a factorized 
form in Eq. (16). We may identify $G(\psi, x)$ as the $x-z$ projection of a general Gaussian distribution that describes the dependence on the angle between $\vec{p}_{1}$ and $\vec{p}_{2}$ in $3 \mathrm{D}$ geometry. In the small width approximation, it can be shown that the corresponding projection onto the transverse, $x-y$, plane, gives a Gaussian form, $G(\Delta \phi, x)$, for the $\Delta \phi$ dependence.

Returning to Eq. (20) which is written for the plane containing $\vec{k}$ and $\hat{z}$, we now write the double differential in both $\Delta \eta$ and $\Delta \phi$, as for the pedestal term, but now for the peak term arising from the $(\mathcal{T S})_{13}(\mathcal{T S})_{24}$ component

$$
\begin{aligned}
\frac{d N_{\pi^{+} \pi^{-}}^{\text {peak }}}{d p_{1} d p_{2} d \eta_{1} d \Delta \eta d \Delta \phi}= & \frac{1}{\left(p_{1} p_{2}\right)^{2}} \xi \sum_{i} \int d k k f_{i}(k) \int d q_{1} \int d q_{2} \theta\left(k-q_{1}-q_{2}\right) \\
& \sum_{j j^{\prime}} \mathcal{T}\left(p_{1}-q_{1}\right)\left\{S_{i}^{j}\left(\frac{q_{1}}{k}\right), S_{i}^{j^{\prime}}\left(\frac{q_{2}}{k-q_{1}}\right)\right\} \mathcal{T}\left(p_{2}-q_{2}\right) \\
& \left.G\left(\psi, \frac{q_{2}}{k}\right)\right|_{\psi=\tan ^{-1} g\left(\eta, \eta_{1}\right)} b G\left(\Delta \phi, \frac{q_{2}}{k}\right),
\end{aligned}
$$

where $b$ is a numerical normalization factor such that when Eq. (42) is integrated over $\Delta \phi$ from -0.5 to +0.5 (the experimental window for projection to the $\Delta \eta$ dependence) one gets

$$
b \int_{-0.5}^{0.5} d \Delta \phi G(\Delta \phi, x=0)=1
$$

in the small $x$ approximation.

Identifying Eq. (42) as $N(1,2)^{\text {peak }}$, we can substitute it into the numerator of Eq. (29) and obtain $d N^{\text {peak }} / d \Delta \eta d \Delta \phi$. There is only one free parameters to adjust; it is $\sigma_{0}$ in Eq. (17). The projections of the double differential distribution to $\Delta \eta$ and $\Delta \phi$ separately can be compared with the data when the pedestal component is added. That is

$$
\begin{aligned}
& \frac{d N^{A P}}{d \Delta \eta}=\int_{-0.5}^{0,5} d \Delta \phi\left[\frac{d N^{\text {ped }}}{d \Delta \eta d \Delta \phi}+\frac{d N^{\text {peak }}}{d \Delta \eta d \Delta \phi}\right] \\
& \frac{d N^{A P}}{d \Delta \phi}=\int_{-1}^{1} d \Delta \eta\left[\frac{d N^{p e d}}{d \Delta \eta d \Delta \phi}+\frac{d N^{\text {peak }}}{d \Delta \eta d \Delta \phi}\right]
\end{aligned}
$$

The pedestal contributions to the above integrals are given by Eqs. (37) and (39), respectively. It should be noted that the latter has a $\Delta \phi$ dependence described by $H(\Delta \phi)$, which is a Gaussian with a half-width $\sigma_{\phi}$ given by Eq. (40). We shall find below that the half-width of the peak term, controlled by $\sigma_{0}$, is much smaller than $\sigma_{\phi}$, so the last terms in Eqs. (44) and (45) dominate the peak structure of the APD. The term $d N^{p e d} / d \Delta \eta d \Delta \phi$ gives rise to a ridge, whose projections are the flat pedestal in $d N^{A P} / d \Delta \eta$, and a broad mount in $d N^{A P} / d \Delta \phi$.

After we put all the pieces together, using Eq. (29) as the important link between our calculation and the measurable quantities, the results from Eqs. (44) and (45) can be compared to data with $\sigma_{0}$ adjusted to fit the peak width. Figure 1 shows the APD in $\Delta \eta$. The solid line is the result of our calculation when we set

$$
\sigma_{0}=0.22 \text {. }
$$


Evidently, it reproduces the data [3] satisfactorily, both in the peak structure and the pedestal. It should be emphasized that although the width and the pedestal height are adjusted to fit by our choice of $\sigma_{0}$ and $\Delta T$, the height of the peak is a consequence of our very complicated calculation involving multiple integrals over many terms based on the recombination model. It is by no means trivial that the data can be fitted so well. In point of fact, we have only calculated $\pi^{+} \pi^{-}$production, whereas the data are for all charged particles. Thus the precise values of the parameters are not as significant as the overall situation where the pedestal and peak structure of the $\Delta \eta$ distribution of the data can be reproduced by our description of dihadron correlation.

Turning now to the APD in $\Delta \phi$, we have no free parameters to adjust, since the double differentials in the square brackets in Eqs. (44) and (45) are identical. Upon integration over $\Delta \eta$ we obtain the solid line in Fig. 2, which compares well with the data [3]. The dashed line indicates the pedestal contribution from Eq. (39). It plays the role of the flat pedestal in Fig. 1, but here it vanishes at $|\Delta \phi|=1$ because of the subtraction scheme. Since the peak structure in $\Delta \phi$ is much narrower than the broad mount of the pedestal (due to $\sigma_{0} \ll \sigma_{\phi}$ ), we have applied the vanishing condition at $|\Delta \phi|=1$ only to the $d N^{\text {ped }} / d \Delta \phi$ component, knowing that the $d N^{\text {peak }} / d \Delta \phi$ component is negligible at the wings of the peak.

Figures 1 and 2 represent our main results on dihadron correlation in central $\mathrm{Au}+\mathrm{Au}$ collisions at $\sqrt{s}=200 \mathrm{GeV}$, when the trigger particle is kept within the range $4<p_{\text {trig }}<6$ $\mathrm{GeV} / \mathrm{c}$ and the associated particle in the range $2<p_{\text {assoc }}<4 \mathrm{GeV} / \mathrm{c}$. We can readily calculate the APD at higher momentum ranges, but not at lower momenta, since our model is not reliable for parton momentum less than $1 \mathrm{GeV} / \mathrm{c}$. For that reason we do not calculate the APD for $0.15<p_{\text {assoc }}<4 \mathrm{GeV} / \mathrm{c}$, even though data for that range are presented in [3].

\section{Conclusion}

To summarize, we have successfully reproduced the data that show the existence of peaks in $\Delta \eta$ and $\Delta \phi$ distributions, the former sitting above a flat pedestal, while the latter sitting above a broad mount. Some parameters are used to fit the data, but the essence of our work is not data fitting. We have demonstrated that the physics underlying the detail structure of the jet characteristics observed in RHIC experiments can be understood in the framework of parton recombination. Jets produced in heavy-ion collisions create shower partons that are in the environment of thermal partons, which can themselves be enhanced by the passage of hard partons through the medium. That view is probably shared by all theoretical approaches to the problem. The issue then is how those partons hadronize. Different models treat the hadronization process differently. Our approach emphasizes the recombination of the thermal partons and the shower partons in the intermediate $p_{T}$ region. That is our way of accounting for the medium effects on jets. So far we have not encountered any obstacle in understanding the data in that way. Data fitting is only a concrete demonstration that the details of the jet structure can be quantitatively reproduced in our treatment. The hadronization formalism is thereby enriched by the determination of some features in the model by phenomenology.

Our first discovery in this work is that the pedestal in the $\Delta \eta$ distribution can be related to $\Delta T$ in the local thermal distribution. No "long-range correlation" has been put in by 
hand. The pedestal is a consequence of the recombination of the thermal partons among themselves, which are only indirectly affected by hard scattering through the enhancement of $T$ due to energy loss. Thus the pedestal is not a part of the jet, but cannot be present without a jet. This chain of successive connections involving energy loss, enhanced thermal partons, their hadronization by recombination, and the elevated APD in $\Delta \eta$ is certainly very different from a model where an explicit correlation is put in by hand.

Our second achievement in this work is the success in describing the peak structures in both $\Delta \eta$ and $\Delta \phi$ distributions in terms of one angular distribution of the shower partons in a jet cone. No "short-range correlation" has been put in by hand. The only primitive correlation in the problem is that which exists among the shower partons in a jet. The properties of that correlation in the momentum magnitudes of the shower partons have already been described in [12]. Here we show how the correlations manifest themselves in the angular variables of the produced pions.

There is one caveat in our analysis that should be noted. We have calculated only $\pi^{+} \pi^{-}$ production, whereas the data are on all charged hadrons. Thus numerically the parameters determined here are not definitive. Since particle identification is steadily being improved, the more appropriate arena for detailed matching of theory and experiment is when the data for the production of specific species become available.

Despite the tentativeness of the value of $\Delta T$ given in Eq. (38) on account of the statement made just above, our assertion that the pedestal is related to the enhancement of $T$ of the thermal partons remains unaffected. As a test of that assertion we propose that the proton associated with a $\pi^{+}$trigger be measured in addition to the measurement of a $\pi^{+}$or $\pi^{-}$ associated particle. The $p / \pi$ ratio of the associated particles in a $\pi^{+}$triggered jet should be a good probe of the physical mechanism underlying both the pedestal and the peak. The recombination approach to the problem is well positioned to calculate that ratio, as it has for single-particle distribution [9]. We surmise that the pedestal part of the $p / \pi$ ratio would be higher than in the single-particle case because $\Delta T>0$.

What we have done in this paper is influenced greatly by our intention to understand the pedestal and peak structure in the data of [3]. As a consequence, our analysis involves many integrals that correspond to the experimental cuts in the data. Having determined the origin of the pedestal and peaks in the $\Delta \eta$ and $\Delta \phi$ distributions, we are now equipped to launch a study of the correlation problem independent of any triggers and related experimental cuts, on which more and more data are becoming available.

\section{Acknowledgment}

We have benefitted from discussions with X. N. Wang and C. B. Yang in the preliminary stage of this work. Communication with F. Wang has also been helpful. This work was supported, in part, by the U. S. Department of Energy under Grant No. DE-FG03-96ER40972. 


\section{References}

[1] For reviews see M. Gyulassy and L. McLerran, Nucl. Phys. A 750, 30 (2005).

[2] P. Jacobs and X. N. Wang, Prog. Part. Nucl. Phys. 54, 443 (2005).

[3] J. Adams et. al., (STAR Collaboration), nucl-ex/0501016.

[4] C. A. Gagliardi (for STAR Collaboration), Proceedings of Hard Probes 04, Ericeira, Portugal (2004).

[5] D. Magestro (for STAR Collaboration), Proceedings of Hard Probes 04, Ericeira, Portugal (2004).

[6] Y. Guo (for STAR Collaboration), nucl-ex/0502015.

[7] B. Jacak (for PHENIX Collaboration), Proceedings of Hard Probes 04, Ericeira, Portugal (2004).

[8] J. Adams et al. (STAR Collaboration), nucl-ex/0411003.

[9] R. C. Hwa and C. B. Yang, Phys. Rev. C 70, 024905 (2004).

[10] R. C. Hwa and C. B. Yang, Phys. Rev. Lett. 93, 082302 (04).

[11] R. C. Hwa and C. B. Yang, Phys. Rev. C 70, 054902 (2004).

[12] R. C. Hwa and Z. Tan, nucl-th/0503052.

[13] R. C. Hwa and C. B. Yang, Phys. Rev. C 66, 025205 (2002).

[14] R. C. Hwa and C. B. Yang, Phys. Rev. C 70, 024904 (2004).

\section{Figure Captions}

Fig. 1. Associated particle distribution in $\Delta \eta$ for $2<p_{T}<4 \mathrm{GeV} / \mathrm{c}$ with trigger particle in $4<p_{T}^{\text {trig }}<6 \mathrm{GeV} / \mathrm{c}$. The data from Ref. [3] are for all charged hadrons in the respective $p_{T}$ ranges. The solid line is the result of our calculation of $\pi^{-}$associated with $\pi^{+}$trigger.

Fig. 2. Same as in Fig. 1 except that the distribution is in $\Delta \phi$. The dashed line represents the pedestal effect in $\Delta \phi$ forced to vanish at $|\Delta \phi|=1$ by the subtraction scheme discussed in Sec. 3. 


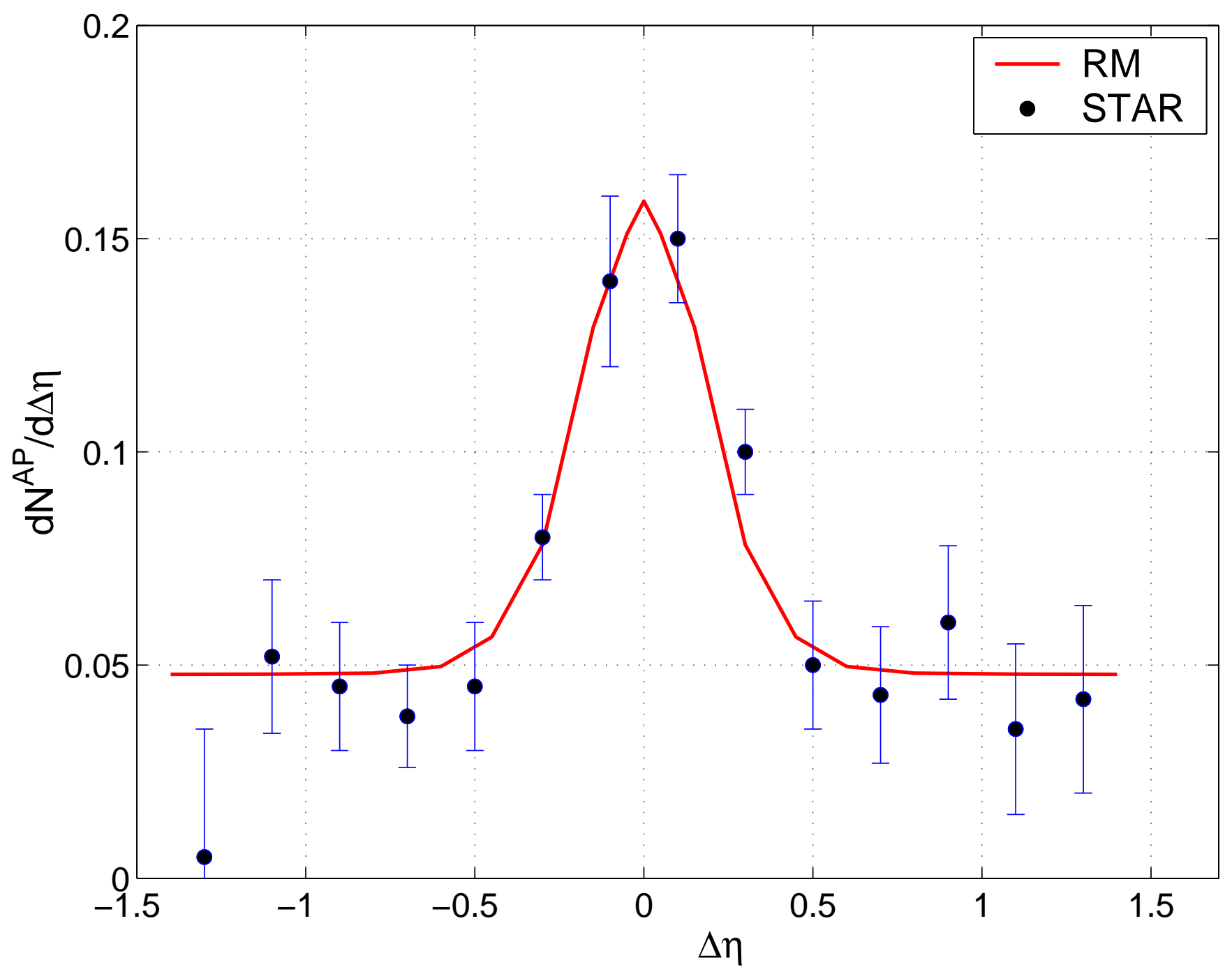




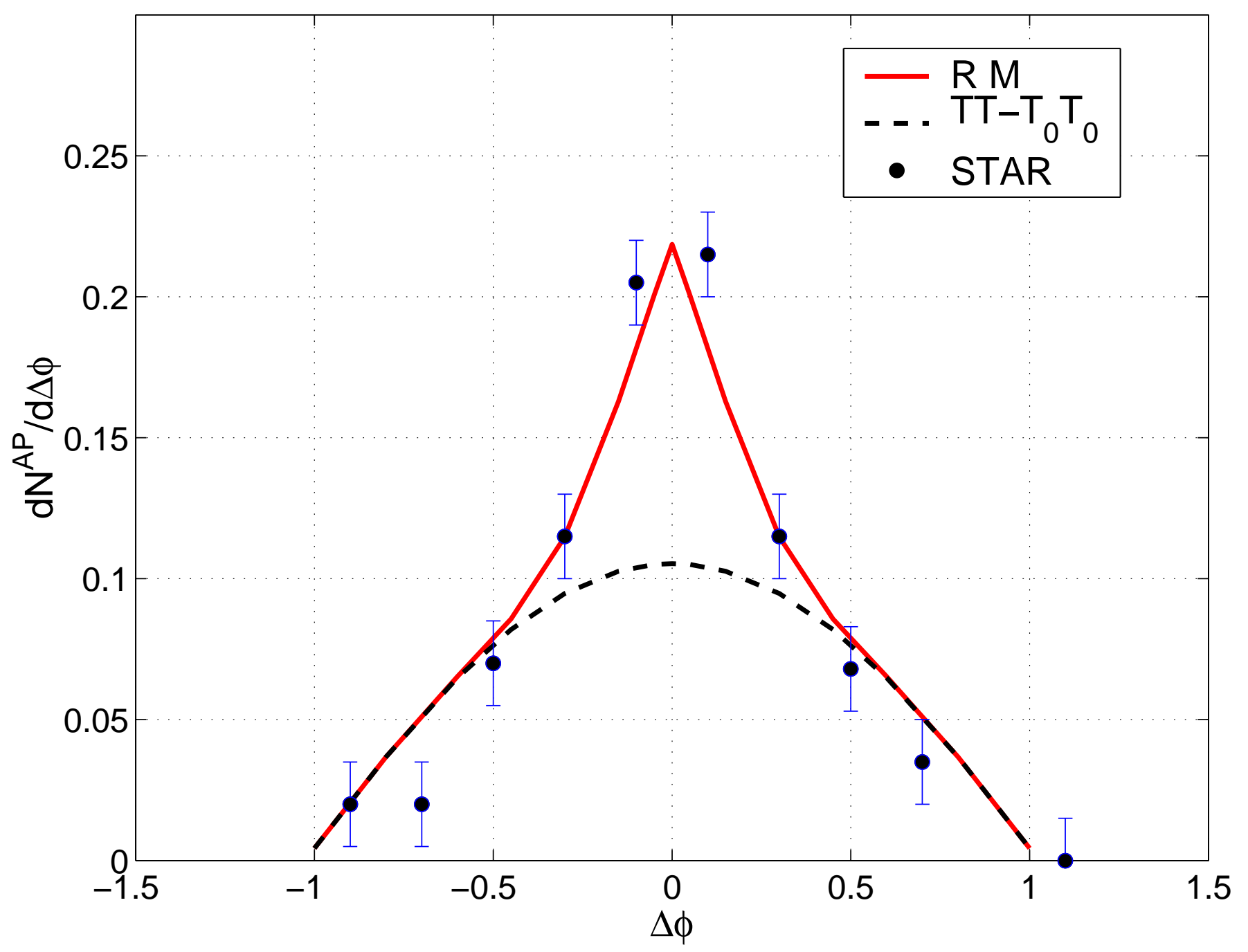

